\title{
Phase Response Curves, Delays and Synchronization in MATLAB
}

\author{
W. Govaerts and B. Sautois \\ Department of Applied Mathematics and Computer Science, Ghent University, \\ Krijgslaan 281-S9, B-9000 Ghent, Belgium \\ \{willy.govaerts, bart.sautois\}@ugent.be
}

\begin{abstract}
MatCont is a Matlab software package for the study of dynamical systems. We extended this package with the functionality of computing the phase response curve or PRC of a neural model. An important application lies in the study of synchronization of neurons. In synchronization studies, often the delays in a network are discarded or set to zero. We show in this paper that the delay can be crucial for the ability of neurons in an excitatory network to synchronize. We also show that, by studying the PRC of a neural model, one can compute the necessary delay to allow synchronization or phase-locking in a network of such neurons.
\end{abstract}

\section{Introduction}

Cl_MatCont [1] and its GUI version MatCont 2] are Matlab packages for the study of dynamical systems and their bifurcations. Among other things, they support the numerical continuation of equilibria, limit cycles, limit points, Hopf points, fold, flip, and torus bifurcations of cycles. Both packages are freely available at http://www.matcont.ugent.be/. The MATLAB platform, for which the packages are written, is attractive for both developer and user. The problem of speed, which arises since MATLAB is not compiled, is solved by incorporating C-code, while preserving the program's portability and user-friendly features.

In neural modeling, a phase response curve is a function that indicates the response of a spiking neuron to an input pulse. A positive value means that the next spike will be fired sooner, a negative value means that the next spike is delayed. In CL_MATCONT and MATCONT we have incorporated the functionality of calculating the phase response curve $(\mathrm{PRC})$ and its derivative $(\mathrm{dPRC})$ of limit cycles during continuation.

In the present paper, we show the importance of the PRC and $\mathrm{dPRC}$ for synchronization and phase-locking properties of neurons in a network with excitatory connections. In studies of such networks, very often delays are regarded as being of less importance, or simply set to zero $([3,4])$. We show that in fact, the delays of connections play a crucial role in the ability of neurons to phase-lock or synchronize. This necessary delay can be computed using the PRC.

We restrict ourselves to networks of identical neurons, with uniform excitatory connections. More chaotic synchronization studies have been made, using other software, e.g. in Mathematica [5, 6]. 


\section{The MATLAB Software}

CL_MatCont and MatCont are packages for the study of dynamical systems and their bifurcations. They are successor packages to packages written in compiled languages (Fortran, C, C++). The full Matlab platform of CL_MatConT and MatCont is attractive because it makes them more user-friendly than other packages, and portable to all operating systems, as MATLAB is platformindependent. Also, MATLAB has GUI-development tools built-in and allows a standard handling of data files, graphical output, etcetera. A typical screenshot, showing several of the windows that can be opened in MATCONT, is shown in Figure 1.

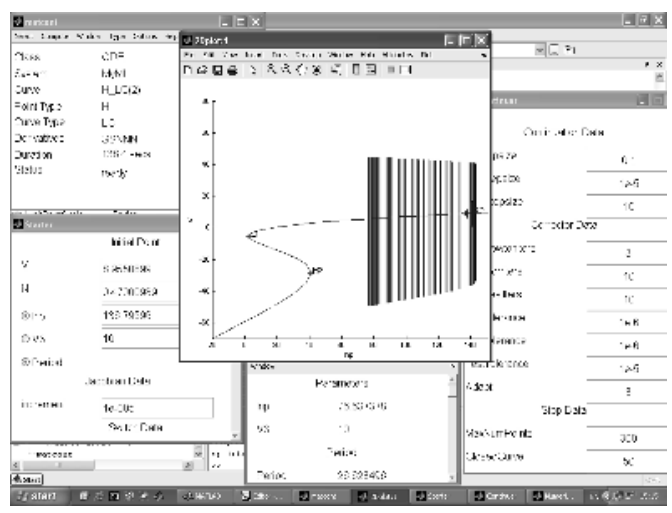

Fig. 1. Typical screenshot from MATCONT

MatCont uses the MATLAB standard ODE-solvers for time-integration of dynamical systems. Also, it makes intensive use of the linear algebra routines that form the core of MATLAB. Further, it uses the sparse solver routines that are provided in MATLAB.

On the other hand, the code is inevitably slower than a compiled programming language. It is possible however, through some intermediate C-code, to have the $\mathrm{C}$-code and MATLAB-code communicate with each other. Because there is a Ccompiler present in every version of MATLAB, we can supply the C-source-code and let the program compile the C-code at runtime. This causes a slight delay at startup of the program, negligible when compared to the time needed to do further computations. The advantage of this approach is that it keeps the program completely platform-independent. The platform-dependent compiled $\mathrm{C}$-code is only generated at runtime, and is thus adapted to the machine.

\section{Phase Response Curve of a Model}

The phase response curve or PRC of a periodically spiking neuron expresses its reaction to an input pulse given at a certain time during its cycle period. 

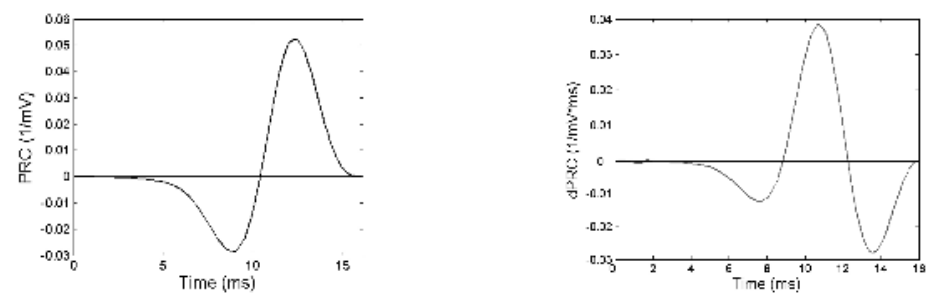

Fig. 2. Left: PRC $(\phi)$ for the Hodgkin-Huxley system. Right: dPRC $\left(\phi^{\prime}\right)$ for the same system. The equations and parameter values for this model are given in [7].

More formally, the phase response curve is a function, which is defined over the timespan of one cycle in the spike train of a firing neuron with no extra input. As starting point of the period we take the peak of a spike. At each point of the cycle, the phase response curve (PRC) indicates the effect on the oncoming spikes of an input pulse to the neuron at that particular time in its cycle.

If we denote by $T_{\text {old }}$ the period of the unperturbed cycle, and by $T_{n e w}$ the period when the input pulse $\alpha$ is given at time $t$, the linearized phase response curve is defined by

$$
\begin{aligned}
\operatorname{PRC}(t) & =\phi(t)=\lim _{\alpha \rightarrow 0} \frac{G(t, \alpha)}{\alpha}, \\
G(t, \alpha) & =\frac{T_{\text {old }}-T_{\text {new }}(\alpha)}{T_{\text {old }}} .
\end{aligned}
$$

Note that time $t$ is expressed in ms, and the input pulse $\alpha$ in $\mathrm{mV}$. Thus, $G(t, \alpha)$ is dimensionless, and the actual PRC, $\phi$, has units $1 / \mathrm{mV}$.

It is also possible to compute the derivative of the PRC $\left(\mathrm{dPRC}\right.$ or $\left.\phi^{\prime}\right)$, which can be useful, as we show in this paper. This function has units $1 / \mathrm{mV}^{*} \mathrm{~ms}$. An example of both curves is given in Figure 2. For a more detailed discussion of linearized PRCs and how we compute them using our software package MATCONT, we refer to [7].

\section{1:1 Phase-Locking to Repeated Inputs}

Consider an oscillator with period $T$, which gets repeated external inputs with a fixed period which is close to $T$, say $T_{\Delta}$. The ability of the oscillator to phase-lock with this input is an important concept, as discussed in [8] and [9].

Say the oscillator or neural model fires a spike at time $t=0$, and gets an input with strength $\alpha$ at time $t=t_{1}$. Then the next spike of the neuron will occur at time $t=T-\alpha T \phi\left(t_{1}\right)$. If we call $t_{2}$ the distance between the following spike and input, then

$$
t_{2}=\left(t_{1}+T_{\Delta}\right)-T\left(1-\alpha \phi\left(t_{1}\right)\right)
$$

So

$$
t_{2}=\left(t_{1}+T_{\Delta}\right)+\alpha T \phi\left(t_{1}\right) \bmod T
$$




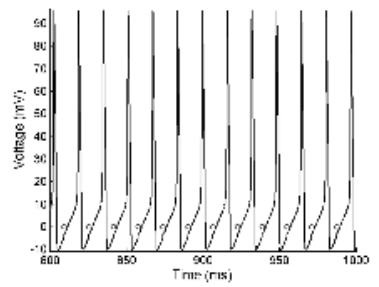

Fig. 3. The spikes show the spiking regime of the Hodgkin-Huxley neuron. The circles indicate when the repetitive input comes in. It is clear that this is a phase-locked state, for $t_{1} \approx 7 \mathrm{~ms}$, with period $16.28 \mathrm{~ms}$.

Any fixed point of this map has the property that

$$
T_{\Delta}+\alpha T \phi\left(t_{1}\right)=0 \bmod T
$$

Thus, in such points, 1:1 phase locking is possible. Since the linearized PRC of a neuron can only be used reliably for $\alpha$ small enough, the period of the inputs is not too different from the oscillator's original period.

The formula can be understood as follows: when the PRC for a certain delay is positive (negative), then the next spikes will be sped up (delayed), therefore there can only be synchronization if $T_{\Delta}<T\left(T_{\Delta}>T\right)$.

The so obtained fixed point of the map $t_{1} \rightarrow t_{1}+T_{\Delta}+\alpha T \phi\left(t_{1}\right)-T$ is stable if and only if its unique multiplier $1+\alpha T \phi^{\prime}\left(t_{1}\right)$ has modulus less than 1 , i.e.

$$
\frac{-2}{\alpha T}<\phi^{\prime}\left(t_{1}\right)<0
$$

So, if an oscillator gets a series of inputs of which the period is close to its own original period $\left(T_{\Delta}\right.$ satisfies (4) ), and the delay $t_{1}$ meets constraint (5), then one can get stable synchronization of the oscillator to the inputs.

These results were tested on the Hodgkin-Huxley equations, with inputs of strength 1, for which the PRC and dPRC are shown in Figure 2 For an input with a longer period, both $\phi$ and $\phi^{\prime}$ will have to be negative at the input time to allow phase-locking, so the inputs will come earlier than about $8.5 \mathrm{~ms}$ after the last spike. In all cases with negative $\phi$ and negative $\phi^{\prime}, \phi^{\prime}>-2 / T=-0.125$, so constraint (5) is always satisfied. And finally, the exact timing of input and spike at the phase-locked state will depend on the period of the spike. Since $T_{\Delta}>T$, we know that $T_{\Delta}-T=T \phi\left(t_{1}\right)$. E.g. when we give inputs with period $T_{\Delta}=T(1+0.01) \approx 16.28$, the neuron will always phase-lock with the inputs in such a timing that the inputs will reach the neuron at time $t_{1}$, such that $\phi\left(t_{1}\right)=-0.01$, and in the negative regime of $\phi^{\prime}$, this is the case for $t_{1}=6.747$ ms. The experimental results are shown in Figure 3, with circles indicating the times of arriving input. An analogous analysis can be done for inputs arriving with a shorter period than that of the uncoupled model. 


\section{Two Single-Spiking Neurons}

\subsection{Synchronization}

Consider two identical neurons with excitatory connections to each other, and suppose that the connection has a delay of duration $t_{1}$ and a connection strength of size $\alpha$. Suppose the neurons, without input, have period $T$, and the linearized PRC of the neurons is given by the function $\phi$. Finally assume that neuron 1 fires at time $t=0$, and neuron 2 at time $t=\Delta, \Delta<t_{1}$.

Then neuron 1 gets an input signal at time $\Delta+t_{1}$ and neuron 2 gets a signal at absolute time $t_{1}$, which is in relative time, relative to its spike, $t_{1}-\Delta$ (due to the phase difference). For the next spikes we conclude:

- neuron 1 will fire its next spike at $t=T-\alpha T \phi\left(t_{1}+\Delta\right)$.

- neuron 2 will fire its next spike at $t=(\Delta+T)-\alpha T \phi\left(t_{1}-\Delta\right)$.

The time difference map (for the time difference between spikes of the two neurons)

$$
\Delta \rightarrow \Delta+\alpha T \phi\left(t_{1}+\Delta\right)-\alpha T \phi\left(t_{1}-\Delta\right)
$$

has the trivial fixed point $\Delta=0$ which is stable if and only if its unique multiplier $1+2 \alpha \phi^{\prime}\left(t_{1}\right)$ has modulus less than 1, i.e.

$$
\frac{-1}{\alpha T}<\phi^{\prime}\left(t_{1}\right)<0
$$

Once the neurons are synchronized, the new period is $T-\alpha T \phi\left(t_{1}\right)$.

This shows that for two identical neurons with excitatory connections to synchronize, the connection delay plays a crucial role. Moreover, the delays that allow synchronization, can be determined from the period of the uncoupled neuron, its PRC and its dPRC.

Consider two identical Hodgkin-Huxley neurons, connected through excitatory connections with strength 1 . From the plot of $\phi^{\prime}$ in Figure2, we notice that $\phi^{\prime}\left(t_{1}\right)$ is always greater than $-1 / T \approx-0.0624$. So constraint (7) is always met when $\phi^{\prime}\left(t_{1}\right)<0$. So whenever we initiate the neurons with a connection delay $t_{1}$ such that $\alpha \leq 1, \phi^{\prime}\left(t_{1}\right)<0$, the neurons will move to synchronization with new period $T-\alpha T \phi\left(t_{1}\right)$.

An example is shown in Figure 4. The first plot shows the start, and the second one the result, when starting with $2 \mathrm{HH}$-neurons, firing $1 \mathrm{~ms}$ apart, and with a connection delay of $6 \mathrm{~ms}$. They clearly converge, with a new period of $16.1 \mathrm{~ms}$, which matches our formula.

Analogously, experiments support the theory that there is synchronization of the two neurons with a shorter emerging period when $\phi^{\prime}\left(t_{1}\right)<0$ and $\phi\left(t_{1}\right)>0$, and there is no synchronization if $\phi^{\prime}\left(t_{1}\right)>0$.

\section{$5.2 \quad$ 1:1 Phase-Locking}

If a neuron has a phase response curve $\phi$ such that $\phi\left(t_{1}+\Delta\right)=\phi\left(t_{1}-\Delta\right)$, for a certain connection delay $t_{1}$ and a phase difference $\Delta$, then this point is a fixed point of the map (6). 

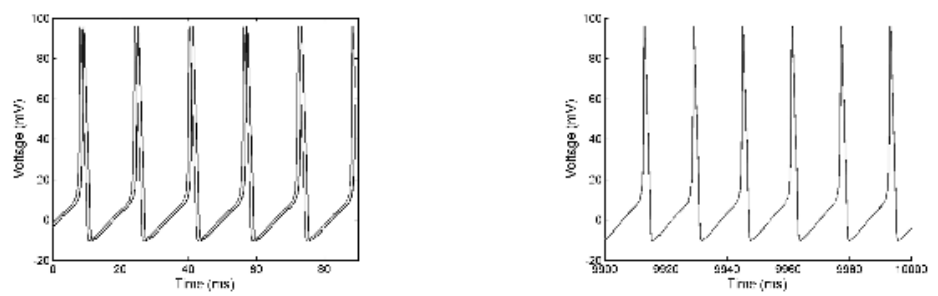

Fig. 4. Left: 2 HH-neurons spiking with $1 \mathrm{~ms}$ difference in timing. Right: The 2 neurons have converged.
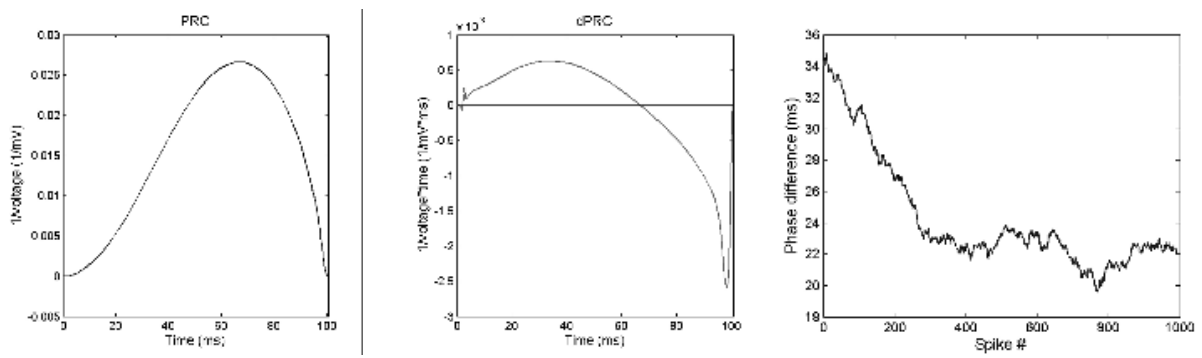

Fig. 5. Left: PRC for the Connor system. Middle: dPRC for the same system. Right: phase difference between two spiking neurons with excitatory connection, evolving to a stable state.

This fixed point is stable when

$$
\begin{array}{r}
\left|1+\alpha T \phi^{\prime}\left(t_{1}+\Delta\right)+\alpha T \phi^{\prime}\left(t_{1}-\Delta\right)\right|<1 \\
\frac{-2}{\alpha T}<\phi^{\prime}\left(t_{1}+\Delta\right)+\phi^{\prime}\left(t_{1}-\Delta\right)<0
\end{array}
$$

So the fall of $\phi$ must be steeper than its rise.

As an example, this is the case in the Connor model. The equations for this model are given in [10]. Figure[5] shows the PRC (left) and dPRC (middle) of the Connor model at parametervalue $I=8.49$. When the connection delay $t_{1}=64$, then $\Delta \approx 22$ is a fixed point, such that $\phi\left(t_{1}+\Delta\right)=\phi\left(t_{1}-\Delta\right)=0.019$. Also $\phi^{\prime}\left(t_{1}+\Delta\right)=-8 e-4$ and $\phi^{\prime}\left(t_{1}-\Delta\right)=6 e-4$, so constraint (8) is met. A result is shown in the rightmost picture in Figure 5 it shows how the phase difference between two spiking neurons moves from its initial value, chosen at $35 \mathrm{~ms}$, to 22 ms, the stable fixed point.

\section{Synchronization of an N-Neuron Single-Spiking Network}

It is possible to generalize the theory for a 2-neuron network to an N-neuron network. Consider a network of $\mathrm{N}$ identical oscillators with period $T$ and PRC $\phi$. Suppose this is an all-to-all connected excitatory network, with all connections having a delay $t_{1}$ and strength $\alpha$. 
Let $X=\left(x_{1} x_{2} \ldots x_{N}\right)^{T}$ be a vector of time variables close to each other such that oscillator 1 spikes at time $x_{1}$, oscillator 2 at time $x_{2}$, etc. Then the next spikes will be fired at times

$$
A(X)=\left(\begin{array}{l}
x_{1}+T-\alpha T \phi\left(t_{1}+x_{2}-x_{1}\right)-\ldots-\alpha T \phi\left(t_{1}+x_{N}-x_{1}\right) \\
x_{2}+T-\alpha T \phi\left(t_{1}+x_{1}-x_{2}\right)-\ldots-\alpha T \phi\left(t_{1}+x_{N}-x_{2}\right) \\
\ldots \\
x_{N}+T-\alpha T \phi\left(t_{1}+x_{1}-x_{N}\right)-\ldots-\alpha T \phi\left(t_{1}+x_{N-1}-x_{N}\right)
\end{array}\right)
$$

If there is synchronization, i.e. $x_{1}=x_{2}=\ldots=x_{N}$, then all components of $A(X)$ are equal to $x_{1}+T-\alpha(N-1) T \phi\left(t_{1}\right)$. So the period of the synchronized state must be $T-\alpha(N-1) T \phi\left(t_{1}\right)$.

We want to know whether this synchronized state is stable and attracting. Consider the map

$$
F: X \mapsto A(X)-\left(\begin{array}{c}
T\left(1-\alpha(N-1) \phi\left(t_{1}\right)\right) \\
T\left(1-\alpha(N-1) \phi\left(t_{1}\right)\right) \\
\cdots \\
T\left(1-\alpha(N-1) \phi\left(t_{1}\right)\right)
\end{array}\right)
$$

$F$ has a fixed point $(0 \ldots 0)^{T}$ and its Jacobian $F_{X}$ evaluated there is

$$
\left(\begin{array}{cccc}
1+\alpha T \phi^{\prime}\left(t_{1}\right)(N-1) & -\alpha T \phi^{\prime}\left(t_{1}\right) & \ldots & -\alpha T \phi^{\prime}\left(t_{1}\right) \\
-\alpha T \phi^{\prime}\left(t_{1}\right) & 1+\alpha T \phi^{\prime}\left(t_{1}\right)(N-1) & \ldots & -\alpha T \phi^{\prime}\left(t_{1}\right) \\
\ldots & \ldots & \ldots & \ldots \\
-\alpha T \phi^{\prime}\left(t_{1}\right) & \ldots & \ldots 1+\alpha T \phi^{\prime}\left(t_{1}\right)(N-1)
\end{array}\right)
$$

To determine the asymptotic stability of the synchronized state, we consider the difference vector $Y=\left(y_{2} \ldots y_{N}\right)^{T}=\left(x_{2}-x_{1} \ldots x_{N}-x_{1}\right)^{T}$.

The difference vector of $F(X)$ is $G(Y)=\left(\begin{array}{llll}F_{2}(X)-F_{1}(X) & \ldots & F_{N}(X)-\end{array}\right.$ $\left.F_{1}(X)\right)^{T}$ where $F_{i}$ is the $i$ - th component of $F$.

So the stability of the synchronized state is determined by the map $G$. Its Jacobian $G_{Y}$ can easily be obtained from $F_{X}$ and is given by

$$
\left(\begin{array}{ccc}
1+\alpha T \phi^{\prime}\left(t_{1}\right)(N-1) & \ldots & -\alpha T \phi^{\prime}\left(t_{1}\right) \\
\ldots & \ldots & \ldots \\
-\alpha T \phi^{\prime}\left(t_{1}\right) & \ldots & 1+\alpha T \phi^{\prime}\left(t_{1}\right)(N-1)
\end{array}\right)+\left(\begin{array}{ccc}
\alpha T \phi^{\prime}\left(t_{1}\right) & \ldots & \alpha T \phi^{\prime}\left(t_{1}\right) \\
\ldots & \ldots & \ldots \\
\alpha T \phi^{\prime}\left(t_{1}\right) & \ldots & \alpha T \phi^{\prime}\left(t_{1}\right)
\end{array}\right)
$$

This is a diagonal matrix with $(N-1)$ equal diagonal elements and thus all eigenvalues equal to $1+\alpha N T \phi^{\prime}\left(t_{1}\right)$. So the synchronization is asymptotically stable if

$$
\frac{-2}{\alpha N T}<\phi^{\prime}\left(t_{1}\right)<0
$$

This is the generalization of constraint (7) to the synchronization of an Nneuron network with all-to-all excitatory connections.

This is again confirmed by tests on a network of Hodgkin-Huxley neurons. Figure 6] shows on the left the initial state: $8 \mathrm{HH}$ neurons, firing spikes $1 \mathrm{~ms}$ 

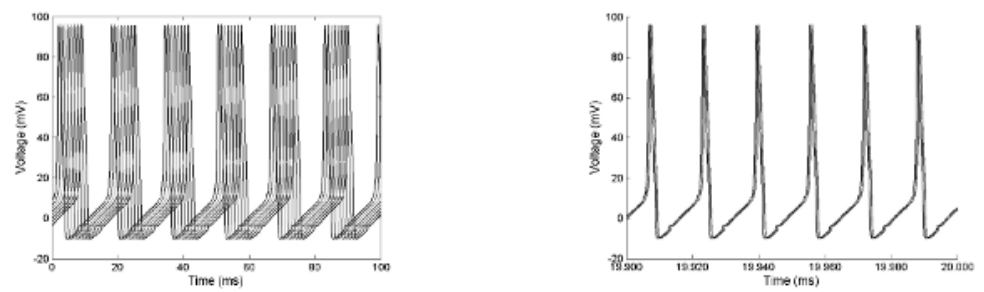

Fig. 6. Left: 8 HH-neurons spiking with $1 \mathrm{~ms}$ difference in timing. Right: The 8 neurons have converged.

apart. They are connected through excitatory connections, giving each other pulses of size $1(\mathrm{mV})$. The connections have a delay of $6 \mathrm{~ms}$. After $20000 \mathrm{~ms}$, the result is shown on the right of the figure: all 8 neurons have synchronized strongly.

Acknowledgement. Bart Sautois is a research assistant of the Research Foundation - Flanders (FWO - Vlaanderen).

\section{References}

1. Dhooge, A., Govaerts, W., Kuznetsov, Yu.A., Mestrom, W., Riet, A.M.: A continuation toolbox in MATLAB. Manual (2003), http://www.matcont.ugent. be/doc_cl_matcont.zip

2. Dhooge, A., Govaerts, W., and Kuznetsov, Yu.A.: MatCont: A matlab package for numerical bifurcation analysis of ODEs. ACM TOMS 29,2 (2003) 141-164

3. Hansel, D., Mato, G., Meunier, C.: Synchrony in excitatory neural networks. Neural Comput. 7 (1995) 307-337

4. van Vreeswijk, C., Hansel, D.: Patterns of synchrony in neural networks with spike adaptation. Neural Comput. 13 (2001) 959-992

5. Gutiérrez, J.M., Iglesias, A.: A Mathematica package for the analysis and control of chaos in nonlinear systems. Comp. Phys. 12,6 (1998) 608-619

6. Iglesias, A., Galvez, A.: Revisiting some control schemes for chaotic synchronization with Mathematica. LNCS 3516 (2005) 651-658

7. Govaerts, W., Sautois, B.: Computation of the phase response curve: a direct numerical approach. To appear in Neural Comput. 18,4 (2006)

8. Brown, E., Moehlis, J., Holmes, P.: On the phase reduction and response dynamics of neural oscillator populations. Neural Comput. 16 (2004) 673-715

9. Izhikevich, E.M.: Dynamical systems in neuroscience: the geometry of excitability and bursting. To be published by the MIT press (2006)

10. Connor, J.A., Walter, D., McKown, R.: Modifications of the Hodgkin-Huxley axon suggested by experimental results from crustacean axons. Biophys. J. 18 (1977) 81-102 